\title{
ASSESSING THE IMPACT OF “BRAIN TRAINING” ON DRIVING PERFORMANCE, VISUAL BEHAVIOR, AND NEUROPSYCHOLOGICAL MEASURES
}

\author{
Jonathan Dobres ${ }^{1}$, Anya Potter ${ }^{1,2}$, Bryan Reimer ${ }^{1}$, Bruce Mehler ${ }^{1}$, Alea Mehler ${ }^{1}$ \& \\ Joseph Coughlin ${ }^{1}$ \\ ${ }^{1}$ The Massachusetts Institute of Technology, AgeLab, Cambridge, MA, USA \\ ${ }^{2}$ University of Massachusetts, Boston, MA, USA \\ Email: jdobres@mit.edu
}

\begin{abstract}
Summary: As the population has become both older and more technologically literate, a new class of "brain training" computer programs have gained in popularity. Though these programs have attracted substantial attention from scientists and consumers, the extent of their benefits, if any, remain unclear. Here we employ neuropsychological tests and behavioral metrics collected during periods of real-world driving (with and without manipulations of cognitive load) to evaluate the effects of training with Posit Science's DriveSharp software. We find that DriveSharp's training effects appear in in-lab measures of Useful Field of View but did not translate to changes in actual driving performance or changes in visual behavior in consistent or quantifiable ways in the sample assessed. The implications of these results and relevant limitations of the present research are discussed.
\end{abstract}

\section{INTRODUCTION}

Aging is associated with steady declines in a wide variety of cognitive domains, including reduced motor control and slowing of reaction times, memory impairment, and fundamental changes in visual processing (Cabeza et al., 2004; Govenlock, Taylor, Sekuler, \& Bennett, 2009; McIntosh et al., 1999). As we become a demographically older populace, it will be increasingly important to be able to detect, compensate for, and potentially halt or reverse such declines. A substantial body of research has shown that motor, memory, and visual functions in the older population can be greatly improved through regular, concentrated training regimens (Ball \& Sekuler, 1986; R. Sekuler \& Ball, 1986). With the advent of personal computers and portable gaming systems, a new class of “brain training” software has become available, such Nintendo's Brain Age, CogniFit’s ${ }^{\circledR}$ brain training programs, Lumos Labs’ Lumosity ${ }^{\mathrm{TM}}$, NeuroQuest’s CogFit, and Posit Science’s DriveSharp ${ }^{\circledR}$ system. Many of these cognitive interventions focus on improving visual processing and reaction times, domains that are highly relevant to the operation of a motor vehicle. Indeed, Posit Science explicitly advertises the potential benefits of its training system in terms of perception of the roadway (Posit Science, 2012). While such systems have garnered considerable attention from consumers and the popular press, the extent of their actual impact on driver behavior remains unclear.

A major question surrounding these types of cognitive interventions is whether their purported benefits transfer to real-world tasks and situations. A user might improve greatly on the specific tasks presented by the training software, but these improvements will do little good if they do not generalize beyond computer and neuropsychological testing. Previous research has shown that cognitive interventions can lead to improvements on the tasks specific to training (Ball \& 
Sekuler, 1986; Ball et al., 2002), and, while transfer of learning is less common, it has been documented in some limited contexts (Willis et al., 2006). Transfer of learning has also been documented in cases where an enriched training stimulus is used, such as action video games. Training with such games results in improvements in a wide variety of visual functions, though it should be noted that the detailed environments central to these games are unlike the simpler, more easily parameterized tasks presented in many cognitive training packages (Bavelier et al., 2011).

The current study was designed to directly address the effects of Posit Science's DriveSharp training system on real-world driving behavior. Study participants were tested on a number of neuropsychological measures and driving behavior was recorded under varying levels of cognitive demand to establish baseline performance metrics. These measures were repeated after a two-week period in which half of the participants underwent at-home training with DriveSharp. Our goal was to examine what impact this training regimen might have on a group of older, relatively high functioning drivers.

\section{METHODS}

\section{Participants}

A total of 38 subjects (21 males) aged 60-75 took part in the study. Participants were required to be active, relatively healthy, experienced drivers with a valid driver's license for at least the past 3 years, and without history of accident in the last year. Recruitment was carried out in the greater Boston area. The study was approved by MIT's IRB and informed consent was obtained.

One participant failed to engage in the secondary tasks and was excluded from analysis. Two participants' eye tracking data were captured for less than $33 \%$ of the data analysis periods and were also excluded. Three subjects did not return for the follow-up session. This left a total of 32 subjects (17 males). Sixteen subjects were assigned to the intervention group, and another 16 were assigned to the control group. Demographic statistics are summarized in Table 1.

Table 1: Demographic data for the study's participants

\begin{tabular}{llll}
\hline Group & $\mathbf{N}$ & Mean Age (SD) & Total Training Time, mins (SD) \\
\hline Intervention & $16(8$ male $)$ & $66.8(4.5)$ & $500.8(72.9)$ \\
\hline Control & $16(9$ male $)$ & $66.3(5.3)$ & NA \\
\hline
\end{tabular}

\section{Apparatus}

Vehicle data. Vehicle telemetry data were obtained from an instrumented 2010 Lincoln MKS sedan at a rate of $10 \mathrm{~Hz}$. Mean driving speed, steering wheel position, and acceleration data were recorded directly from the vehicle CAN bus, and summary metrics were computed from these data for select driving periods. We computed fine wheel reversal rates from wheel position using a $2^{\circ}$ reversal gap and report the total number of reversals per period of measurement. We computed acceleration events as a combination of longitudinal and lateral acceleration using the Pythagorean theorem. Independent acceleration events were identified as two accelerations greater than $0.1 \mathrm{~g}$ separated by 2 or more seconds (Reimer, Mehler, Wang, \& Coughlin, 2012). 
Eye behavior was logged at $60 \mathrm{~Hz}$ using a faceLAB ${ }^{\circledR} 5.0$ (Seeing Machines, Canberra, Australia) eye tracking system.

Secondary tasks. Subjects performed a clock visualization task (Schlorholtz \& Schieber, 2006) and a 1-back delayed digit recall task (Mehler, Reimer, \& Dusek, 2011) during specific periods of highway driving in each session. In the clock task, subjects are given a time (for instance, “3:40"), and are asked to visualize whether the angle formed by the hands of the clock at that time is less than $90^{\circ}$. In the 1-back task, a sequence of 1-digit numbers is read aloud, and subjects are asked to repeat the digit that immediately preceded the one currently being spoken (see Mehler et al., 2011 for details).

Neuropsychological tests. Subjects participated in a battery of neuropsychological tests. These included the Attention Network Test (ANT) (Fan, McCandliss, Sommer, Raz, \& Posner, 2002) and Useful Field of View (UFOV) test (Ball \& Owsley, 1993). Evaluations were conducted at the start of each driving session prior to the driving tasks.

\section{Driving session procedure}

Participants were trained on the clock visualization and 1-back tasks to ensure proficiency prior to driving. During the course of the experiment, a research associate was seated in the rear of the vehicle to monitor data acquisition and driver safety. The research associate provided directions and answered essential questions during the experiment while minimizing extraneous conversation.

The driving route consisted of approximately 10 minutes of urban driving in the Boston area to reach an interstate highway (I-93 north) and approximately 45 minutes of highway driving followed. Data considered in this analysis were collected during the highway driving portion. On both the northward and southward drives, this included a 2-minute reference period of single task driving (baseline), a 2-minute period of dual-task driving (driving while performing the clock task on the northward drive and the 1-back task on the southward drive), and a 2-minute single task recovery period. The baseline and recovery periods were separated from the dual task periods by 30 seconds. All instructions and tasks were pre-recorded and played automatically during the driving session.

\section{At-home training regimen}

After the first driving session, 16 subjects were given copies of Posit Science's DriveSharp (also known as DriveSharp with InSight) visual training computer program. Over the following two weeks, subjects were asked to practice daily with DriveSharp's “Jewel Diver" and "Road Tour" exercises, which are similar to multiple object tracking and useful field of view tasks, respectively. DriveSharp uses a staircase (Levitt, 1971) to adjust the difficulty of the task to the participant, converging on a $70.7 \%$ correct performance criterion. Posit Science recommends a minimum "dose" of 8 hours (480 minutes) of training. The 16 control subjects simply returned for a follow-up driving session two weeks after the initial one, without any intervening task or training. 


\section{Analysis}

Measures of horizontal and vertical gaze dispersion were calculated for select driving periods by projecting the driver's angle of gaze onto a flat plane at a distance corresponding to the vehicle's windshield and then computing a simple standard deviation of gaze points. This technique has been shown to be sensitive to changes in gaze concentration under cognitive demand (Reimer et al., 2012). Data were analyzed in R (R Core Team, 2012). ANOVAs with repeated measures were conducted for each dependent variable. ANOVA by ranks was employed in cases where data were non-normal. For the intervention group, Pearson correlations between the amount of time spent on the training tasks and improvements in neuropsychological and vehicle behavior measures were also computed. Vehicle telemetry and eye gaze data were analyzed for the 2minute periods of time before, during, and after the clock and 1-back tasks corresponding to baseline, active task, and recovery periods.

\section{RESULTS}

\section{Training tasks}

Intervention group subjects averaged 500.8 minutes of training (SD 73.0), with 10 of 16 subjects completing at least 480 minutes of training (all subjects are included in subsequent analyses regardless of total training time). Subjects trained more on the Road Tour task vs. Jewel Diver (274.8 min vs. $226 \mathrm{~min}, \mathrm{p}=.008$, Wilcoxon signed rank test).

\section{Neuropsychological metrics}

The ANT's reaction time and conflict subscales were considered for analysis (both are measured in milliseconds). Between the first and second evaluation, reaction times decreased by $48.6 \mathrm{msec}$ $\left(\mathrm{F}_{(1,15)}=13.49, \mathrm{p}=.002\right)$ and $25.5 \mathrm{msec}\left(\mathrm{F}_{(1,15)}=3.75, \mathrm{p}=.073\right)$ for the overall reaction time and conflict scale measures, respectively. Improvements did not depend on intervention or its interaction with visit. Improvement on the conflict task within the intervention group was significantly correlated with total time spent training $(r=-.69, \mathrm{p}=.026)$.

The UFOV test (Ball \& Owsley, 1993) has three subscales: processing speed, divided attention, and selective attention. Results are quantified as the presentation time needed to reach a certain staircase criterion (faster presentations indicate stronger performance). For the processing speed task, all subjects performed to criterion at $16.7 \mathrm{~ms}$ (the limit of the screen's refresh rate). On the divided attention task, there was a significant interaction between intervention and visit. The control group's presentation time decreased by $28.0 \mathrm{~ms}$, while the intervention group's decreased by $83.5 \mathrm{~ms}\left(\mathrm{~F}_{(1,15)}=4.59, \mathrm{p}=0.040\right)$. On the selective attention task, there was a significant decrease in threshold of $26.7 \mathrm{msec}$ between the two visits $\left(\mathrm{F}_{(1,15)}=12.08, \mathrm{p}=.002\right)$, as well as a borderline interaction between intervention and visit $\left(\mathrm{F}_{(1,15)}=3.20, \mathrm{p}=.084\right)$. Improvement on the divided attention task was significantly correlated with time spent on the Road Tour training task $(\mathrm{r}=-.51, \mathrm{p}=.044)$. 


\section{Gaze dispersion during highway driving}

The effect of task period was significant for horizontal gaze dispersion $\left(\mathrm{F}_{(2,58)}=54.42, \mathrm{p}<.001\right)$, decreasing from an average standard deviation of $11.3 \mathrm{~cm}$ during the baseline period to $8.6 \mathrm{~cm}$ during the task period, then rebounding to baseline levels. No other factors or interactions (visit, intervention group, type of task) were significant. For vertical dispersion, there was a main effect of period $\left(\mathrm{F}_{(2,58)}=4.72, \mathrm{p}=.01\right)$, with gaze dispersion increasing significantly during the posttask recovery period relative to baseline and task periods $(6.6 \mathrm{~cm}$ during recovery vs. $6.0 \mathrm{~cm}$ at baseline and $6.2 \mathrm{~cm}$ during the task) as well as main effect of task type $\left(\mathrm{F}_{(2,29)}, \mathrm{p}=.003,6.5 \mathrm{~cm}\right.$ during n-back vs. $6.0 \mathrm{~cm}$ during clock task). No other significant effects were found.

\section{Driving performance}

Vehicle telemetry statistics were calculated for three 2-minute periods (baseline, task, and recovery) for both the clock and 1-back tasks. These included mean speed, number of microaccelerations, and number of wheel reversals greater than $2^{\circ}$ during each period.

Measurements of mean speed showed that control subjects drove $4 \mathrm{~m} / \mathrm{sec}$ faster on average than intervention subjects (main effect of group, $\mathrm{F}_{(1,28)}=9.55, \mathrm{p}=.005$ ), that subjects drove $3.4 \mathrm{~m} / \mathrm{sec}$ faster during the clock task compared to the n-back (main effect of task, $\mathrm{F}_{(1,28)}=29.74, \mathrm{p}<$ $.001)$, and that subjects drove $2.6 \mathrm{~m} / \mathrm{sec}$ slower during the recovery period $\left(\mathrm{F}_{(2,56)}=20.90, \mathrm{p}<\right.$ $.001)$. Additionally, there was a significant interaction between task type and period $\left(\mathrm{F}_{(2,56)}=\right.$ $20.24, \mathrm{p}<.001)$, and a borderline interaction between task type and visit $\left(\mathrm{F}_{(2,56)}=3.48, \mathrm{p}=\right.$ .075). Amount of time spent on training was not significantly correlated with changes in vehicle speed.

There were no significant differences for measures of micro-accelerations, aside from a borderline effect of task ( 0.35 accelerations/period during the clock task vs. 0.28 during the nback task, $\left.\mathrm{F}_{(1,28)}=3.47, \mathrm{p}=.073\right)$ and a borderline interaction between task and period $\left(\mathrm{F}_{(2,56)}=\right.$ 2.66, $\mathrm{p}=0.079)$. No significant training time correlations were observed.

For wheel reversals, there was an increase of 1.9 reversals/period at second visit $\left(\mathrm{F}_{(1,28)}=4.91\right.$, $\mathrm{p}$ $=.035)$, as well as a significant main effect of period (1.7 more reversals during the recovery period as compared to baseline and task, $\left.\mathrm{F}_{(2,56)}=3.45, \mathrm{p}=.039\right)$, and a significant interaction between task and period $\left(\mathrm{F}_{(2,56)}=6.77, \mathrm{p}=.002\right)$. Time spent on the Road Tour training task was positively correlated with increased wheel reversals during the clock task $(r=.71, p=.005)$.

\section{DISCUSSION}

The results are consistent with the idea that using the DriveSharp training system produces improvements in UFOV, but the benefits of training did not significantly impact the other neuropsychological measures considered or the measures assessed during driving when compared against a control group. The UFOV divided attention task was the only measure to demonstrate a clear effect of DriveSharp training. We also found that the amount of time spent on the Road Tour training task correlated with improvements on the UFOV test. However, since Road Tour is explicitly modeled on the UFOV task, the degree to which this is evidence of the 
apparent "transfer” between these two tasks should not be overstated. Although horizontal gaze dispersion showed exquisite sensitivity to changes in cognitive demand across task types and differences in vertical dispersion were significantly different between the clock and 1-back tasks, these measures did not reveal any effects related to training.

The overall pattern of results is nebulous and somewhat difficult to interpret. The present study is consistent with previous work showing that DriveSharp training leads to improvements on the UFOV test (Wolinsky et al., 2011). However, the present data do not support the notion that DriveSharp engenders transferrable cognitive benefits, consistent with similar work examining the effects of cognitive training on simulated driving sessions (Gaspar, Neider, \& Simons, 2012). We extend these findings by showing a lack of transfer to an attention related neuropsychological measure (the ANT), gaze behavior, and the real-world driving behavior measures considered here. It is possible that other measures, perhaps an on-road version of the peripheral detection task, might reveal differences in useful field of view characteristics that are not evident in the gaze metrics. Given that there have been reports of a reduction in accidents with UFOV training, other research might productively investigate whether the experience of training leads some individuals to reevaluate their driving risk and self-regulate their driving behavior in a risk reducing manner (i.e. reducing miles driven, reduced night time driving, etc.).

Several systemic factors should be kept in mind when considering these results. First, our sample is relatively small (16 subjects per group). Second, DriveSharp training was self-administered in subject's homes, thus making it difficult to ensure that all subjects were fully invested in the task and trained with it appropriately. Third, our sample was drawn from a volunteer population of older adults that are intellectually curious, more high-functioning, and more educated compared to peers in their age group. It is possible that DriveSharp provides greater benefits to persons who have experienced more pronounced cognitive declines, whereas the intervention provides little or no benefit to higher-functioning adults. This would agree with studies of the Wii Fit exercise system, which show that the system has promise in rehabilitative contexts, particularly for lower-functioning older adults, but provides benefits that are relatively small compared to traditional therapeutic regimens (Bateni, 2012). Lastly, long-lasting changes in cognition and visual processing often require extensive, intensive training. While we do not entirely discount the possibility that this type of intervention could produce such changes, it may be the case that a longer or more intense training period is necessary.

\section{ACKNOWLEDGEMENTS}

We gratefully acknowledge the USDOT’s Region I New England University Transportation Center at MIT and The Santos Family Foundation for support of this project and Posit Science Inc. for academic discounts on the DriveSharp software.

\section{REFERENCES}

Ball, K., \& Owsley, C. (1993). The useful field of view test: a new technique for evaluating agerelated declines in visual function. Journal of the American Optometric Association, 64(1), 7179.

Ball, K., \& Sekuler, R. (1986). Improving visual perception in older observers. Journal of Gerontology, 41(2), 176-182. 
Ball, K., Berch, D. B., Helmers, K. F., Jobe, J. B., Leveck, M. D., Marsiske, M., Morris, J. N., et al. (2002). Effects of cognitive training interventions with older adults: a randomized controlled trial. JAMA: the Journal of the American Medical Association, 288(18), 2271-2281.

Bateni, H. (2012). Changes in balance in older adults based on use of physical therapy vs the Wii Fit gaming system: a preliminary study. Physiotherapy, 98(3), 211-216.

Bavelier, D., Green, C. S., Han, D. H., Renshaw, P. F., Merzenich, M. M., \& Gentile, D. A. (2011). Brains on video games. Nature reviews. Neuroscience, 12(12), 763-768. doi:10.1038/nrn3135

Cabeza, R., Daselaar, S., Dolcos, F., Prince, S., Budde, M., \& Nyberg, L. (2004). Task-independent and task-specific age effects on brain activity during working memory, visual attention and episodic retrieval. Cerebral cortex (New York, NY : 1991), 14(4), 12.

Fan, J., McCandliss, B. D., Sommer, T., Raz, A., \& Posner, M. I. (2002). Testing the efficiency and independence of attentional networks. Journal of cognitive neuroscience, 14(3), 340-347.

Gaspar, J. G., Neider, M. B., \& Simons, D. J. (2012). Examining the Efficacy of Training Interventions in Improving Older Driver Performance. Presented at the Proceedings of the Human Factors and Ergonomics Society Annual Meeting 2012. doi:10.1177/1071181312561007

Govenlock, S. W., Taylor, C. P., Sekuler, A. B., \& Bennett, P. J. (2009). The effect of aging on the orientational selectivity of the human visual system. Vision Research, 49(1), 164-172.

Levitt, H. (1971). Transformed Up-Down Methods in Psychoacoustics. The Journal of the Acoustical Society of America, 49(2B), 467-477.

McIntosh, A., Sekuler, A., Penpeci, C., Rajah, M., Grady, C., \& Bennett, P. (1999). Recruitment of unique neural systems to support visual memory in normal aging, 9(21), 6 .

Mehler, B., Reimer, B., \& Dusek, J. A. (2011). MIT AgeLab Delayed Digit Recall Task(n-back) MIT AgeLab White Paper Number 2011-3B. Massachusetts Institute of Technology, Cambridge, MA. http://agelab.mit.edu/system/files/Mehler_et_al_n-back-white-paper_2011_B.pdf

Posit Science. (n.d.). Posit Science DriveSharp Brain Fitness Exercises. Amazon.com. Retrieved November 14, 2012, from http://www.amazon.com/Posit-Science-DriveSharp-FitnessExercises/dp/B002IFBGPI

R Core Team. (2012). R: A Language and Environment for Statistical Computing. Vienna, Austria. Retrieved from http://www.R-project.org/

Reimer, B., Mehler, B., Wang, Y., \& Coughlin, J. F. (2012). A Field Study on the Impact of Variations in Short-Term Memory Demands on Drivers' Visual Attention and Driving Performance Across Three Age Groups. Human Factors, 54(3), 454-468.

Schlorholtz, B., \& Schieber, F. (2006). Assessment of age differences in mental workload while driving using verbal versus visual-spatial subsidiary tasks, 50(22), 2378-2382.

Sekuler, R., \& Ball, K. (1986). Visual localization: age and practice. Journal of the Optical Society of America. A, Optics and image science, 3(6), 864-867.

Willis, S. L., Tennstedt, S. L., Marsiske, M., Ball, K., Elias, J., Koepke, K. M., Morris, J. N., et al. (2006). Long-term effects of cognitive training on everyday functional outcomes in older adults. JAMA: the Journal of the American Medical Association, 296(23), 2805-2814.

Wolinsky, F. D., Vander Weg, M. W., Howren, M. B., Jones, M. P., Martin, R., Luger, T. M., Duff, K., et al. (2011). Interim analyses from a randomised controlled trial to improve visual processing speed in older adults: the Iowa Healthy and Active Minds Study. BMJ Open, 1(2), e000225-e000225. doi:10.1136/bmjopen-2011-000225 